\title{
Prepro-thyrotropin-releasing hormone 178-199 increases sensitivity of AtT-20 cells to dexamethasone
}

\author{
S Revskoy, J Schwartz ${ }^{\mathbf{1}}$ and E Redei \\ The Asher Center, Department of Psychiatry and Behavioral Sciences, Northwestern University Medical School, Chicago, Illinois 60611, USA \\ ${ }^{1}$ Department of Physiology, University of Adelaide, Adelaide, South Australia 5005, Australia \\ (Requests for offprints should be addressed to S Revskoy)
}

\begin{abstract}
The prepro-thyrotropin-releasing hormone (ppTRH)derived peptide, ppTRH178-199, has been proposed to inhibit ACTH release at the level of the pituitary and attenuate prolactin and behavioral responses to stress as well. The objective of this study was to elucidate a possible link between the effects of ppTRH178-199 and glucocorticoids on the inhibition of ACTH release in corticotrophs. Compared with mock-transfected cells, AtT-20 cells that were stably transfected with full-length ppTRH cDNA showed significantly increased sensitivity to dexamethasone, as measured by inhibition of ACTH release. In a group of control cells, expressing a mutated form of ppTRH cDNA lacking the ppTRH178-199
\end{abstract}

region, sensitivity to dexamethasone was not different from mock-transfected controls. Exogenous ppTRH178199 also increased the inhibitory effect of dexamethasone in wild-type AtT-20 cells. The combined effect of dexamethasone and ppTRH cDNA in cells that express the latter was not due to increased endogenous secretion of ppTRH178-199 in response to dexamethasone, as dexamethasone was independently found to inhibit secretion of ppTRH178-199. Taken together, these data suggest that ppTRH178-199 can interact with the glucocorticoid negative feedback inhibition to regulate ACTH secretion.

Journal of Endocrinology (2001) 171, 491-498

\section{Introduction}

Secretion of adrenocorticotropin (ACTH) by pituitary corticotrophs is subject to positive and negative influences: corticotropin-releasing hormone $(\mathrm{CRH})$ and vasopressin stimulate release of ACTH from the anterior pituitary, while glucocorticoids inhibit ACTH production, as a negative feedback mechanism. Recently, a hypothalamic peptide, prepro-thyrotropin-releasing hormone-derived peptide 178-199 (ppTRH178-199), derived from the rat prepro-thyrotropin-releasing hormone $(\mathrm{ppTRH})$ precursor, has been proposed as a factor that inhibits ACTH secretion in vitro (Redei et al. 1995) and in vivo (McGivern et al. 1997, Engler et al. 1999). In addition, ppTRH178199 has been shown to inhibit stress-induced secretion of prolactin (McGivern et al. 1997) and TRH-induced secretion of growth hormone in rats (Roussel et al. 1994) and chickens (Harvey \& Cogburn 1996). Reports of varying responses of pituitary cells to ppTRH178-199 suggest that the effects of ppTRH178-199 may be more complex than the direct inhibition of ACTH and prolactin secretion.

One potential contributor to the apparently discrepant results with ppTRH178-199 on ACTH secretion (Redei et al. 1995, Nicholson \& Orth 1996) or multiphasic prolactin-secretory patterns in response to the peptide (McGivern \& Handa 1999, Nillni et al. 2001) is the occurrence of interactions between ppTRH178-199 and other regulatory factors. Among the candidate factors likely to interact with ppTRH178-199 are the adrenal steroids.

Glucocorticoids regulate ACTH secretion at multiple levels of the hypothalamic-pituitary-adrenal axis, including the direct inhibition of ACTH synthesis and secretion at the anterior pituitary. Although this regulation is primarily thought to counteract the ACTH secretagogueinduced stimulation in ACTH biosynthesis and secretion, recent evidence obtained in $\mathrm{CRH}$-deficient transgenic mice suggests that peripheral glucocorticoids can regulate pro-opiomelanocortin (POMC) transcription or mRNA stability and production of ACTH separately (Muglia et al. 2000), and further suggests that glucocorticoids also regulate the unstimulated secretion of ACTH. Glucocorticoids act on multiple processes in pituitary cells and are involved in responses in multiple time domains, all of which provide potential sites for interactions with factors other than CRH and vasopressin. Since ppTRH178-199 has been reported to inhibit POMC transcription and ACTH secretion 
(Redei et al. 1995), the possible interaction between its effects and those of glucocorticoids has been explored in the present study.

In this study, we have investigated whether the presence of this peptide affects the dexamethasone-induced inhibition of ACTH release by AtT-20 cells. For this purpose, AtT-20 cells, stably transfected with either the full-length ppTRH cDNA or a mutated ppTRH cDNA lacking the ppTRH178-199 sequence, were treated with dexamethasone and the level of inhibition of ACTH release was determined. In addition, we studied interactions between exogenous ppTRH178-199 and dexamethasone.

\section{Materials and Methods}

\section{Chemicals}

Dexamethasone was obtained from Sigma Chemicals (St Louis, MO, USA). Dulbecco's modified Eagle's medium (DMEM), fetal calf serum (FCS) and antibiotic/ antimycotic mixture were from Gibco Life Technologies (Gaithersburg, MD, USA) and rat ppTRH178-199 was obtained from Peninsula Laboratories (Belmont, CA, USA).

\section{Expression plasmids}

The ppTRH expression plasmids (ppTRH and $\Delta_{\text {aa119-229ppTRH), driven by a human cytomegalovirus }}$ (CMV) immediate early gene promoter, were generated as described previously (Redei et al. 1995). Briefly, the 1322 bp rat ppTRH cDNA (Lee et al. 1988) was subcloned into eukaryotic expression vector pcDNA3 (Invitrogen, San Diego, CA, USA), which contains the aminoglycoside phosphotransferase (neo) gene for G-418 selection. To control for any effects of products of the ppTRH cDNA, aside from those of ppTRH178-199, a mutated form of the gene, $\Delta_{a a 119-229 p p T R H}$, which lacks TRH178-199, was produced by deletion of nucleotides 506-787 in the ppTRH cDNA with BstEII. Both forms of the ppTRH gene, intact and $\Delta_{a a 119-229} \mathrm{ppTRH}$, were transfected into separate populations of AtT-20 cells as described below.

\section{Cell preparation and culture}

AtT-20 cells (AtT-20/D16-16, provided by T Reisine for use in transfections, and S Sabol for effects of exogenous peptides on wild-type cells) were maintained in DMEM containing $10 \% \mathrm{FCS}$ at $37{ }^{\circ} \mathrm{C}$ in an atmosphere of $10 \%$ $\mathrm{CO}_{2}, 90 \%$ air. Initially, three randomly chosen clones of each AtT-20 cell, stably transfected with either ppTRH or $\Delta_{\text {aa119-229 }} \mathrm{ppTRH}$, were analyzed in terms of their baseline ACTH secretion and ppTRH mRNA. Single clones of AtT-20 cells transfected with either construct and demonstrating the highest expression of ppTRH mRNA in Northern analysis (Redei et al. 1995), and a mocktransfected clone, were chosen for further investigation. The experiments were carried out with these stable cell lines which had undergone 18 and 28 passages respectively. To follow the levels of ACTH release, cells were plated at a density of $5 \times 10^{5}$ cells/well in 24-well plates and cultured for 2 days in DMEM containing 10\% FCS.

\section{Experimental protocols}

Effects of ppTRH on ACTH secretion Three sets of cell lines (mock transfected, transfected with ppTRH cDNA and transfected with $\Delta_{a a 119-229} \mathrm{ppTRH}$ ) were incubated for $16 \mathrm{~h}$ in the presence of either complete FCS $(10 \%)$ or $10 \%$ FCS from a batch that was previously stripped by treatment with dextran-coated charcoal (charcoal/dextran-treated FCS; 2\% charcoal and $0 \cdot 2 \%$ dextran mixture). All treatments were carried out in quadruplicate. Supernatants were collected on ice and stored at $-80^{\circ} \mathrm{C}$ until assayed.

Effect of ppTRH on thymidine incorporation Single clones of AtT-20 cells transfected with either construct (selected as described in Materials and Methods) were cultured as described above except that $5 \mu \mathrm{Ci} / \mathrm{ml}{ }^{3} \mathrm{H}-$ thymidine was added to each well at the beginning of a 16-h incubation period. The incorporation of tritium into DNA was determined by liquid scintillation counting (1214 RACKBETA; Perkin Elmer/Wallac, Gaithersburg, MD, USA) after extraction of the DNA by the phenol/ chloroform method followed by ethanol precipitation.

Effect of adsorbable serum factors on expression and secretion of ppTRH In order to determine whether the transfected cells secrete ppTRH178-199 and the potential effects of serum factors on ppTRH expression and secretion, cells were cultured as described above. The cells were incubated for $16 \mathrm{~h}$ in medium containing either complete or charcoal/dextran-treated FCS. At the end of the incubation, media were recovered and frozen at $-80{ }^{\circ} \mathrm{C}$ until analysis by radioimmunoassay. Total RNA was extracted using Trizol reagent, according to the manufacturer's protocol (Gibco Life Technologies). Total RNA concentrations were measured on Beckman spectrophotometer at $260 \mathrm{~nm}$ and each sample was adjusted to a final concentration of $1 \mu \mathrm{g} / \mu \mathrm{l}$. The quantity and quality of RNA were confirmed by gel electrophoresis.

\section{Effect of dexamethasone on secretion of ACTH and} ppTRH ACTH secretion by transfected cells Transfected cells were cultured as described above. The cells were incubated for $16 \mathrm{~h}$ with complete or charcoal/dextrantreated FCS in the absence or presence of varying concentrations of dexamethasone $(1-1000 \mathrm{nM})$. At the end of 
the incubation, media were collected for analysis of the ACTH released by the cells.

ACTH secretion by wild-type AtT-20 cells Non-transfected AtT-20 cells were cultured as described above. They were incubated for $16 \mathrm{~h}$ with complete FCS in the presence and absence of exogenous ppTRH178-199 peptide at 10 or $100 \mathrm{nM}$ and dexamethasone $(1-1000 \mathrm{nM})$. At the end of the incubation, media were collected for analysis of the ACTH released by the cells.

ppTRH178-199 secretion by transfected cells ppTRHtransfected cells were cultured as described above and incubated in the absence and presence of dexamethasone as in the section on ACTH secretion by transfected cells above, except that the maximum concentration of dexamethasone was $100 \mathrm{nM}$. At the end of the 16-h incubation, media were collected for analysis of the ppTRH178-199 released by the cells.

\section{Analyses}

ACTH radioimmunoassay ACTH-like immunoreactivity was measured as previously described (Redei et al. 1995). In brief, ACTH immunoreactivity was assayed using a mid-portion antiserum that recognizes $\mathrm{ACTH}(1-24)$ and $\mathrm{ACTH}(1-39)$ on an equimolar basis. ACTH(1-24) was used as a standard and ${ }^{125} \mathrm{I}-\mathrm{ACTH}$ (1-39) was used as a tracer. The assay sensitivity was $3 \mathrm{pg} /$ tube. Intra-assay and interassay coefficients of variation were $6.4 \%$ and $11.6 \%$ respectively.

ppTRH178-199 radioimmunoassay The radioimmunoassay used in this study for the measurement of ppTRH178-199 was described and validated by Rittenhouse et al. (1996). The tracer was ${ }^{125}$ I-iodinated [Tyr178] ppTRH178-199. Iodination was carried out using the chloramine T method and Sephadex G-10 gel filtration purification. A polyclonal antibody was raised in rabbits against rat ppTRH178-199. The antibody fully crossreacts with ppTRH178-199 and [Tyr178] ppTRH178-199 and with other larger intermediates of the processing from ppTRH to ppTRH178-199 (Nillni et al. 2001), but has no measurable $(<1 \%)$ crossreactivity with any other neuropeptide tested. The working antibody dilution was 1:4000, and the final dilution was 1:12 000; $\mathrm{ED}_{50}$ was $181 \mathrm{pg} /$ tube, using approximately 8000 c.p.m. ${ }^{125}$ I-[Tyr178] ppTRH178-199 in this double antibody assay with a sensitivity of $19 \mathrm{pg} /$ tube.

Northern analysis Northern blotting was carried out on extracted RNA as described previously (Redei et al. 1995). Briefly, total RNA ( $20 \mu \mathrm{g} / \mathrm{lane})$ was electrophoresed in a formaldehyde-denaturing $1 \cdot 2 \%$ agarose gel, transferred to a nylon Hybond-N+ membrane (Amersham Pharmacia Biotech, Piscataway, NJ, USA) and
UV crosslinked to the filter. Filters were prehybridized for $4 \mathrm{~h}$ at $42{ }^{\circ} \mathrm{C}$ with $50 \%$ formamide and $50 \%$ Northern prehybridization solution (Eppendorf 5 Prime, Boulder, CO, USA). Filters were hybridized for $16 \mathrm{~h}$ at $42{ }^{\circ} \mathrm{C}$ in $50 \%$ formamide and 50\% Northern hybridization solution (Eppendorf 5 Prime) with cDNA probes ${ }^{32} \mathrm{P}$-labeled using a random primer labeling kit (Boehringer Mannheim, Indianapolis, IN, USA). The ppTRH probe was the $1322 \mathrm{bp}$ rat ppTRH cDNA insert from a plasmid kindly provided by Dr Stephanie Lee, Tufts University, Boston, MA, USA (Lee et al. 1988). The filters were washed twice for $15 \mathrm{~min}$ each in $2 \times \mathrm{SSC} / 0 \cdot 1 \% \mathrm{SDS}$ at $24^{\circ} \mathrm{C}$, twice for $30 \mathrm{~min}$ each in $0.1 \times \mathrm{SSC} / 0.1 \%$ SDS at $52{ }^{\circ} \mathrm{C}$, and exposed to Kodak X-OMAT-5 film. Densitometry was carried out using an Image Analyzer and Macintosh-based BRAIN system with gray scale calibration. All comparisons were made from RNA samples hybridized on the same filter and normalized to the housekeeping gene glyceraldehyde-3-phosphate dehydrogenase (GAPDH). The GAPDH cDNA was a 983 bp PCR product, generated using primers purchased from Clontech (Palo Alto, CA, USA). Probes were stripped from filters prior to rehybridization by washing in boiling water for $30 \mathrm{~s}$.

\section{Statistics}

Statistical significance was determined by ANOVA followed by $t$-test with Bonferroni's modification. $P<0 \cdot 05$ was considered significant.

\section{Results}

The concentration of cortisol in FCS, as determined by commercial cortisol radioimmunoassay (ICN Biomedicals, Carson, CA, USA), was found to be $56 \mathrm{ng} / \mathrm{ml}$, which is equivalent to $15.4 \mathrm{nM}$ cortisol concentration in the medium that contains 10\% FCS. The charcoal/dextran treatment reduced the cortisol levels of FCS to less than $1 \mathrm{ng} / \mathrm{ml}$.

All transfected cells retained normal morphology and doubling time. Except for transcription and translation of the inserted gene (see below), there were no apparent phenotypic changes to the transfected AtT-20 cells.

\section{Effects of ppTRH on ACTH secretion}

AtT-20 cells transfected with the full-length ppTRH cDNA secreted ACTH at a significantly decreased rate compared with AtT-20 cells transfected with the empty vector (mock transfection). In addition, ACTH secretion by cells transfected with $\Delta_{a a 119-229}$ ppTRH cDNA was indistinguishable from that of mock-transfected cells, consistent with the inhibition being largely due to ppTRH178-199 (Fig. 1). The effect of the full-length ppTRH was more pronounced when comparing secretory 


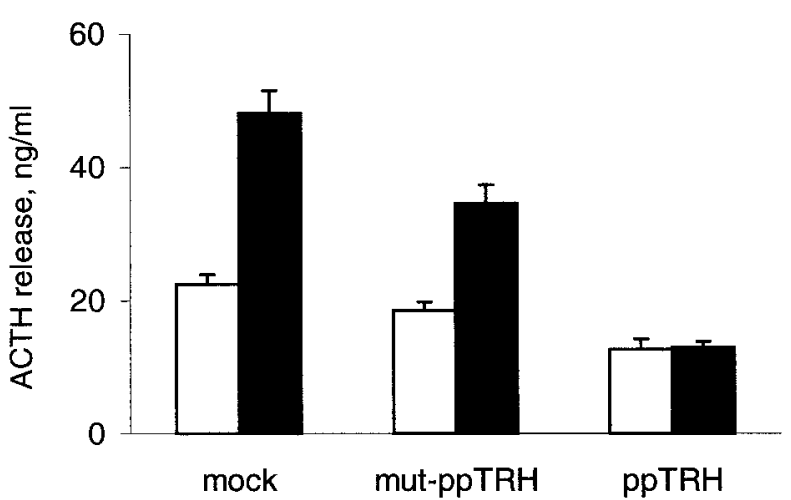

Figure 1 ACTH secretion in AtT-20 cells stably transfected with full-length ppTRH cDNA (ppTRH), vector only (mock) or $\Delta_{\text {aa119-229 }}$ ppTRH (mut-ppTRH). AtT-20 cells were plated at $5 \times 10^{5}$ cells per well containing either complete (open bars) or charcoal/dextran-stripped (solid bars) FCS, incubated for $16 \mathrm{~h}$ and the resultant ACTH secretion measured. The bars represent means \pm S.E.M. from quadruplicate measurements and are representative of those obtained in three separate experiments using different transfected cell lines. The effect of charcoal/ dextran-stripped FCS was increased secretion of ACTH in the mock- (120\%) and $\Delta_{\text {aa } 119-229}$ ppTRH-transfected cells (170\%), but very little or no change $(20 \%)$ in the ppTRH-transfected AtT-20 cells in the other two experiments.

responses of cells incubated with charcoal/dextran-treated FCS. Interestingly, although the removal of steroids and other substances from the FCS by the charcoal/dextran treatment enhanced ACTH release by almost twofold in both mock- and $\Delta_{\text {aa119-22. }} \mathrm{ppTRH-transfected} \mathrm{cells,} \mathrm{there}$ was no effect on AtT-20 cells which expressed the full-length ppTRH cDNA. A virtually identical pattern of decreased ACTH secretion and response to charcoal/ dextran-treated FCS was observed when two other ppTRH-transfected cell-lines were used in comparison with two other $\Delta_{a a 119-229} \mathrm{ppTRH}$ - and mock-transfected AtT20 cell lines.

\section{Effect of ppTRH on thymidine incorporation}

The decreased ACTH secretion observed in AtT-20 cells transfected with ppTRH cDNA was not due to decreased DNA synthesis in general, or to decreased proliferation of these cells during the 16-h incubation. Cell numbers were comparable in the wells of all three transfection groups before and after the 16-h incubation. As expected, ${ }^{3} \mathrm{H}-$ thymidine incorporation was not uniform among the three cell lines (Fig. 2), but incorporation in AtT-20 ppTRH cells was not lower than in the two control groups. In addition, incubation in the presence of charcoal/dextrantreated FCS increased DNA synthesis in both the mock-

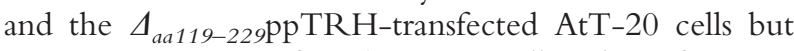
not in ppTRH-transfected AtT-20 cells. This effect was, therefore, similar to that of treated FCS on ACTH secretion (Fig. 1).

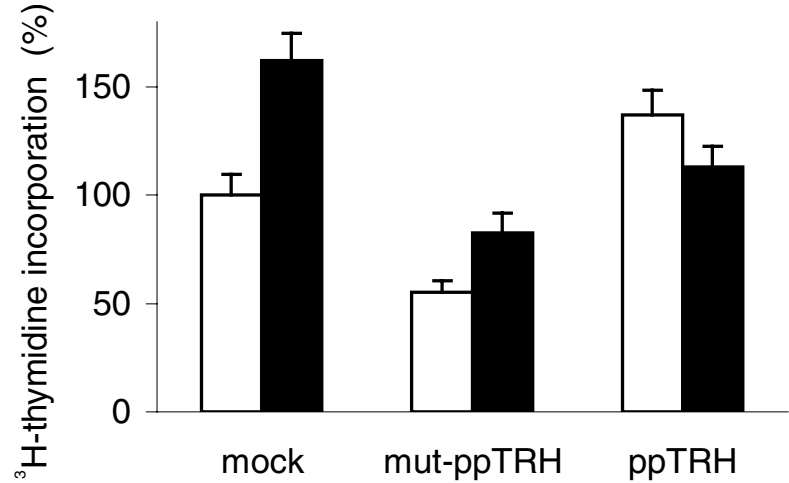

Figure $2{ }^{3} \mathrm{H}$-Thymidine incorporation during a 16-h incubation in stably transfected AtT-20 cells. See legend to Fig. 1. Data are expressed as means \pm S.E.M. of \% change of two independent experiments. ${ }^{3} \mathrm{H}$-Thymidine incorporation into mock-transfected cells incubated in FCS is chosen as $100 \%$.

Effect of adsorbable serum factors on expression and secretion of ppTRH

Removal of steroids by using the charcoal/dextran-treated FCS had no effect on the expression of ppTRH mRNA in the ppTRH cDNA-transfected AtT-20 cells (Fig. 3). This finding was not unexpected since ppTRH transcription was driven by the CMV promoter. In contrast, the rate of ppTRH178-199 release per cell was doubled by the substitution of complete for treated serum during the 16-h incubation. Thus, it seems that there are steroids or other compounds present in complete FCS which normally stimulate secretion of ppTRH178-199.

\section{Effect of dexamethasone on secretion of ACTH by transfected} cells

The secretion rate of ACTH was decreased by dexamethasone at all concentrations $(1-1000 \mathrm{nM}$ for $16 \mathrm{~h})$ in

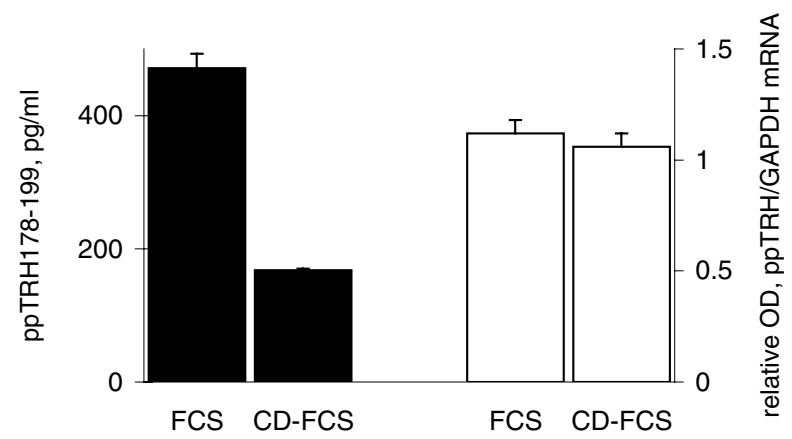

Figure 3 ppTRH178-199 secretion and ppTRH mRNA levels in AtT-20 cells stably transfected with ppTRH cDNA. Cells were incubated for $16 \mathrm{~h}$, ppTRH178-199 concentration was measured and Northern blotting was carried out as described in Materials and Methods. ppTRH mRNA was normalized to GAPDH mRNA and expressed as relative optical density (OD) ratio. CD-FCS, charcoal/dextran-treated FCS. The bars represent the means \pm S.E.M. from quadruplicate measurements and are representative of those obtained in three separate experiments. 


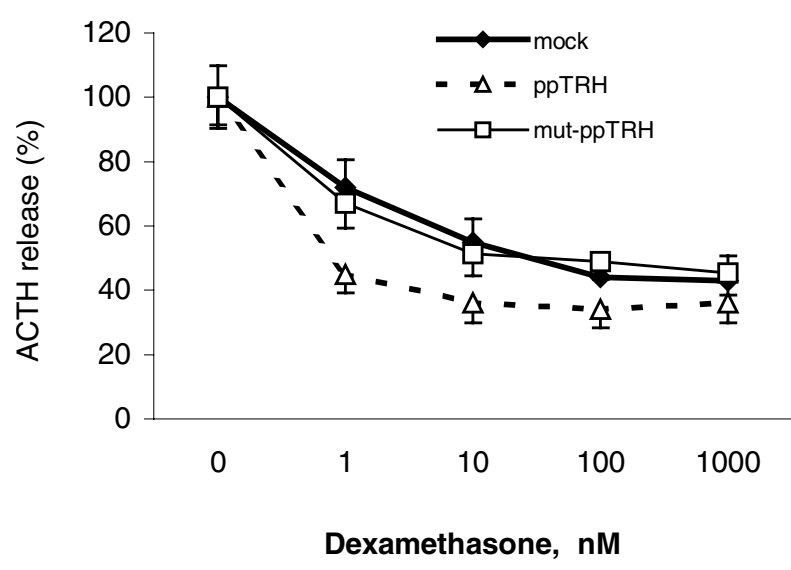

Figure 4 Effect of dexamethasone on ACTH secretion in ppTRH-, $\Delta_{\text {aa119-229 }}$ ppTRH (mut-ppTRH)- and mock-transfected (mock) AtT-20 cells. AtT-20 cells were plated at $5 \times 10^{5}$ cells per well and incubated for $16 \mathrm{~h}$ in the presence of dexamethasone $(0-1000 \mathrm{nM})$ and $10 \%$ FCS. ACTH secretion is expressed as \% change from ACTH levels in the absence of dexamethasone. The results shown are the means \pm S.E.M. of three separate experiments. If not shown, error bars lie within the symbol.

all three cell lines (Fig. 4). Most interestingly, cells transfected with the full-length ppTRH cDNA demonstrated significantly higher sensitivity to dexamethasone than $\Delta_{a a 119-229} \mathrm{ppTRH}$-transfected or mock-transfected cells. While dexamethasone at $1 \mathrm{nM}$ inhibited ACTH secretion in mock-transfected and $\Delta_{a a 119-229} \mathrm{ppTRH}-$ transfected cells by $20-30 \%$, the same concentration of dexamethasone inhibited ACTH secretion by more than $50 \%$ in ppTRH-expressing cells. The difference in sensitivity to dexamethasone between ppTRH-transfected and the $\Delta_{\text {aa119-229 }} \mathrm{ppTRH}$-transfected or mock-transfected AtT-20 cells remained the same in the presence of charcoal/dextran-treated FCS (data not shown).

\section{Effect of dexamethasone and ppTRH178-199 on ACTH secretion by wild-type AtT-20 cells}

In addition to assessing the interaction of dexamethasone with endogenously expressed ppTRH-derived peptides within AtT-20 cells, we tested the direct effect of exogenous ppTRH178-199 on dexamethasone-induced inhib ition of ACTH secretion in wild-type AtT-20 cells. In this experiment, ppTRH178-199 by itself was not effective on basal ACTH secretion. As in the experiments with transfected and mock-transfected AtT-20 cells, incubation of wild-type AtT-20 cells with dexamethasone (1$1000 \mathrm{nM}$ ) for $16 \mathrm{~h}$ in complete FCS produced a saturable inhibition of ACTH secretion, which reached a nadir of ACTH release at $10 \mathrm{nM}$ dexamethasone. When $100 \mathrm{nM}$ ppTRH178-199 was included in the incubation medium, the nadir of ACTH secretion was reached with dexamethasone at $1 \mathrm{nM}$, a concentration that was largely without effect in the absence of ppTRH178-199 (Fig. 5).

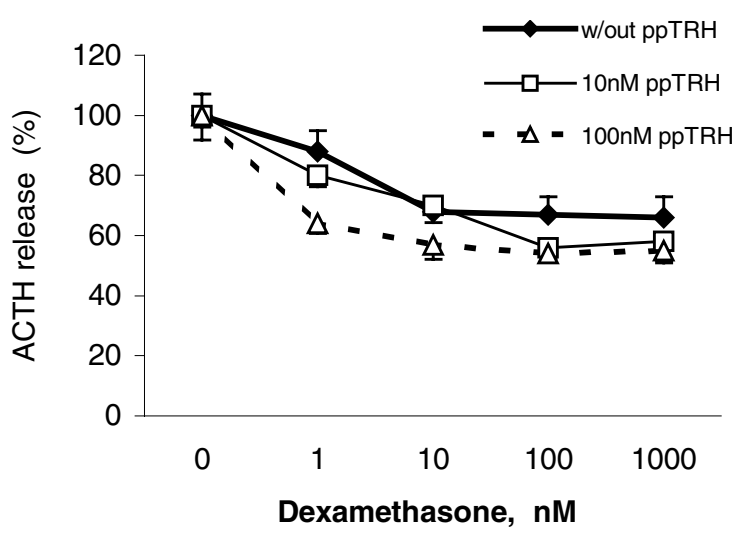

Figure 5 ppTRH178-199 increases dexamethasone-induced inhibition of ACTH release in AtT-20 cells. Wild-type AtT-20 cells were incubated for $16 \mathrm{~h}$ in the presence of $10 \%$ FCS and increasing concentrations of dexamethasone (0-1000 nM) either without (w/out ppTRH) or with $10 \mathrm{nM}$ or $100 \mathrm{nM}$ ppTRH178-199 $(\mathrm{ppTRH})$. The results shown are the means \pm S.E.M. of three separate experiments. If not shown, error bars lie within the symbol.

Interestingly, the concentration-response relationship of ACTH to dexamethasone in wild-type cells in the presence of $100 \mathrm{nM}$ ppTRH178-199 (Fig. 5) is virtually identical to the response to dexamethasone alone in transfected cells that provide their own ppTRH178-199 (Fig. 4). The lower $10 \mathrm{nM}$ dose of ppTRH178-199 had minimal effect on the ACTH response to dexamethasone.

\section{Effect of dexamethasone on ppTRH178-199 secretion by transfected cells}

Given the observation that some factor(s) present in complete serum but not charcoal/dextran-treated serum apparently stimulates secretion of ppTRH178-199, it seemed reasonable to hypothesize that adrenal steroids might play such a role. In this way, the inhibitory effects of glucocorticoids on corticotrophs might be augmented by a secondary effect on ppTRH. To test this possibility, AtT-20 cells stably transfected with ppTRH cDNA were incubated with varying concentrations of dexamethasone (Fig. 6). As in Fig. 3, in the absence of dexamethasone cells incubated with complete FCS secreted more ppTRH178199 than those incubated with charcoal/dextran-treated FCS (data not shown). Surprisingly however, rather than an increase, dexamethasone caused an inhibition of ppTRH178-199 release in both complete and charcoal/ dextran-treated FCS. Dexamethasone $(10 \mathrm{nM})$ inhibited the rate of ppTRH178-199 secretion by $40-50 \%$.

\section{Discussion}

There is now a substantial body of evidence that ppTRH178-199 has potent biological activity ranging 


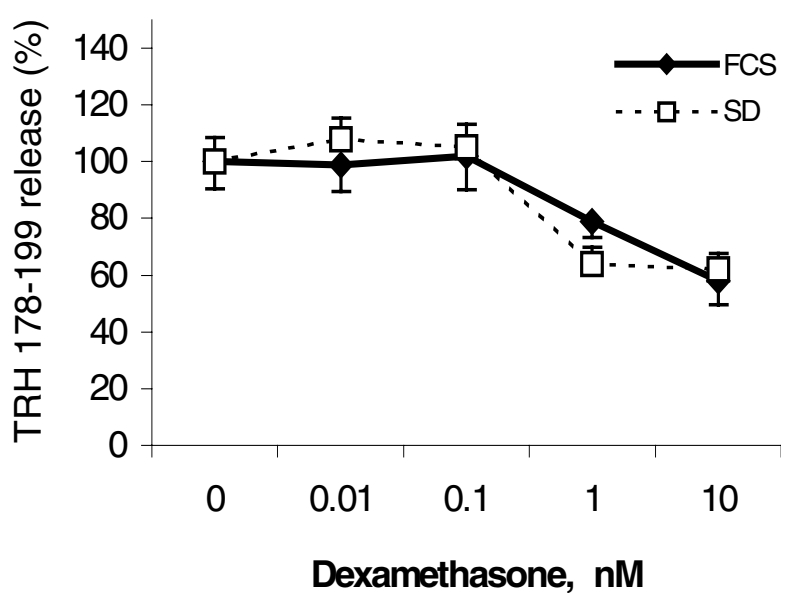

Figure 6 Dexamethasone inhibits secretion of ppTRH178-199 (TRH 178-199) in AtT-20 cells stably transfected with ppTRH cDNA. Cells were incubated for $16 \mathrm{~h}$ in medium containing either complete (FCS) or charcoal/dextran-treated (SD) FCS. The results shown are the means \pm S.E.M. of three separate experiments.

from modulation of secretion of ACTH and prolactin to behavioral effects (Redei et al. 1995, 1999, McGivern et al. 1997, Borlongan et al. 1999, Nillni et al. 2001). Considering the inhibitory effect of ppTRH178-199 on the ACTH stress response, it is important to delineate the physiological role of this peptide in ACTH regulation; particularly to determine whether ppTRH178-199 interacts with glucocorticoids. We addressed this question in an in vitro model, where endogenous secretion of ppTRH178-199 was achieved by using AtT-20 cells stably transfected with either full-length or mutated (lacking the coding region for amino acids 178-199) rat ppTRH cDNA (Redei et al. 1995). We reasoned that if some of the effects of ppTRH178-199 are mediated by augmenting the response of these cells to glucocorticoids, a more efficient inhibition of ACTH secretion could be achieved in cells expressing this peptide.

The present results indicate that the transfected cells did, in fact, secrete ppTRH178-199, and secreted quantitatively less ACTH per cell than either mock-transfected cells or cells transfected with a mutant ppTRH construct lacking the sequence that codes for ppTRH178-199. Given the observed effects of exogenously added ppTRH178-199 on ACTH secretion, it is not unreasonable to expect that the diminished ACTH secretion was a result of the presence of endogenously generated ppTRH178-199.

In addition to the effect of expressing ppTRH178-199 on ACTH secretion by itself, a major finding of this study was that the ACTH-inhibitory response to dexamethasone occurred at lesser concentrations of the steroid in cells that produce ppTRH177-198. Two groups of cells served as controls. One group was phenotypically no different from wild-type AtT-20 cells, having undergone only mock transfection. The other group expressed mutated ppTRH ( $\Delta_{\text {aa119-229ppTRH). Significantly, the latter control pro- }}$ duced responses indistinguishable from those of the mocktransfected cells; expression of ppTRH without the ppTRH178-199 sequence had no effect on the response to dexamethasone. Therefore, the component of the gene product that interacts with the steroid is most likely ppTRH178-199. Consistent with this thought is the observation that incubation of wild-type AtT-20 cells in the presence of exogenous ppTRH178-199 peptide also resulted in an increased sensitivity to dexamethasone as ACTH secretion was inhibited at a lower dose of dexamethasone. Although the nature of the interplay between dexamethasone and ppTRH178-199 was beyond the scope of these studies, the present findings suggest a complex interaction between ppTRH178-199 and dexamethasone on ACTH secretion.

The concentration of ppTRH178-199 secreted into the medium of ppTRH-transfected cells is between 60-200 pM, while the increased sensitivity to dexamethasone was only observed at the $100 \mathrm{nM}$ ppTRH178199 dose in wild-type AtT-20 cells. At first glance this might appear to argue against a physiological role for locally produced ppTRH178-199 in the present experiments. However, the action of ppTRH178-199 might occur at lower concentrations if exposure time is extended. Cells that express ppTRH can secrete ppTRH178-199 constitutively, replacing the rapidly inactivated peptide. As such, prolonged exposure to ppTRH178-199 may have a priming effect. Alternatively, the concentrations of ppTRH178-199 achieved in the immediate vicinity of the secreting cells would likely be many times higher than the average concentration in the incubation medium. This would be physiologically important if the ppTRH178-199 was acting in a paracrine or autocrine manner.

Removal of steroids and other adsorbable factors from the incubation medium by using charcoal/dextran-treated FCS resulted in differential effects, depending on the presence of endogenous ppTRH-derived peptides, including ppTRH178-199. In mock-transfected cells and other transfected cells that had no endogenous ppTRH178-199, removal of serum factors increased DNA synthesis and ACTH secretion. This finding is not unexpected since steroids are among the factors removed from serum by charcoal/dextran treatment, and glucocorticoids are potent inhibitors of ACTH secretion. In contrast, cells that did express ppTRH cDNA and secreted ppTRH178-199 were oblivious to the presence or absence of adsorbable factors. The reasons for this were not readily apparent in the initial stages of the study. The subsequent experiments on the effect of dexamethasone on the secretion of ppTRH178-199 may help provide some explanation. We observed a decrease in secretion 
of ppTRH178-199 in the presence of dexamethasone, consistent with earlier studies of Bruhn et al. (1998), who made a similar observation, despite a stimulatory effect of dexamethasone on ppTRH mRNA and precursor protein. If glucocorticoids inhibit secretion of ppTRH178-199, then the removal of glucocorticoids would allow increased secretion of ppTRH178-199, potentially assuming the modulatory role on ACTH secretion of the steroids. Thus, there would be little or no difference in ACTH secretion by ppTRH-expressing cells between conditions with complete and treated serum. If this is so in the present studies, the virtually identical secretion rates would suggest a very tightly regulated system. Alternatively, it cannot be ruled out that treatment with charcoal/dextran also removed other factors, whose action on ACTH might not be apparent in cells in which ACTH secretion is tonically inhibited, as appears to be the case with ppTRH-expressing cells. Along these lines, it is noteworthy that charcoal/dextran treatment of FCS is known to remove, in addition to steroid hormones, fatty acids and up to $60 \%$ of thyroid hormones (Armelin et al. 1974).

The inhibition of basal, unstimulated ACTH release by dexamethasone was observed during a 16-h incubation of AtT-20 cells. Over that length of time, dexamethasone can inhibit not only the secretion, but also the synthesis of ACTH. In this in vitro system it would be very difficult to dissect the dexamethasone-induced inhibition of ACTH secretion from the effects of dexamethasone on ACTH biosynthesis, since short-term dexamethasone-induced inhibition can only be observed in stimulated ACTH secretion (Woods et al. 1992, Horiba et al. 1993). Thus, these studies cannot answer the question whether endogenous or exogenous ppTRH178-199 increases sensitivity to dexamethasone by modifying secretory processes or by decreasing the releasable pool of ACTH. However, the present data clearly demonstrate an interaction of the product(s) of the ppTRH gene, possibly ppTRH178-199, with dexamethasone in the process of inhibiting ACTH secretion.

In summary, the present results show that the expression and secretion of ppTRH178-199 by transfected corticotrophs causes inhibition of ACTH secretion. The interactions between ppTRH178-199 and glucocorticoids is apparently quite complex, with the latter also having an inhibitory effect on secretion of the former. Since ppTRH178-199 augments the ACTH-inhibitory effect of glucocorticoids, it is tempting to speculate that the interaction may represent redundancy in tissues, such as the anterior pituitary, where ppTRH is expressed (Bruhn et al. 1991) and may act locally to inhibit ACTH secretion. Such a system would enable very precise regulation of ACTH secretion in cases where, for example, input from the hypothalamus is compromised. In any event, such interactions may help explain some of the variability that has been observed in the responses of anterior pituitary cell model systems to glucocorticoids and to ppTRH-derived peptides.

\section{Acknowledgements}

This work was supported by a National Alliance for Research of Schizophrenia and Depression grant (S R).

\section{References}

Armelin HA, Nishikawa K \& Sato GH 1974 Control of mammalian cell growth in culture: the action of protein and steroid hormones as effector substances. In Control of Proliferation in Animal Cells, pp 97-104. Eds B Clarkson \& R Baserga. Cold Spring Harbor: Cold Spring Harbor Laboratory.

Borlongan CV, Stahl CE, Redei E \& Wang Y 1999 Prepro-thyrotropinreleasing hormone 178-199 exerts partial protection against cerebral ischemia in adult rats. Neuroreport 10 3501-3505.

Bruhn TO, Bolduc TG, Maclean DB \& Jackson IMD 1991 ProTRH peptides are synthesized and secreted by anterior pituitary cells in long term culture. Endocrinology 129 556-558.

Bruhn TO, Rondeel JM \& Jackson IM 1998 Thyrotropin-releasing hormone gene expression in the anterior pituitary. IV. Evidence for paracrine and autocrine regulation. Endocrinology 139 3416-3422.

Engler D, Redei E \& Kola I 1999 The corticotropin-release inhibitory factor hypothesis: a review of the evidence for the existence of inhibitory as well as stimulatory hypophysiotropic regulation of adrenocorticotropin secretion and biosynthesis. Endocrine Reviews 20 460-500.

Harvey S \& Cogburn LA 1996 Cryptic peptides of prepro-TRH antagonize TRH-induced $\mathrm{GH}$ secretion in chickens at extrapituitary sites. Journal of Endocrinology 151 359-364.

Horiba N, Nicholson WE, Ch'ng JL \& Orth DN 1993 Chromogranin A does not mediate glucocorticoid inhibition of adrenocorticotropin secretion. Endocrinology 132 1585-1592.

Lee SL, Stewart K \& Goodman RH 1988 Structure of the gene encoding rat thyrotropin releasing hormone. Journal of Biological Chemistry 263 16604-16609.

McGivern RF \& Handa RJ 1999 PreproTRH178-199 is a modulator of anterior pituitary prolactin secretion. Program of the 81st Annual Meeting of the Endocrine Society, San Diego, CA, p 203.

McGivern RF, Rittenhouse P, Aird F, Van de Kar LD \& Redei E 1997 Inhibition of stress-induced neuroendocrine and behavioral responses in the rat by prepro-thyrotropin-releasing hormone 178-199. Journal of Neuroscience 17 4886-4894.

Muglia LJ, Jacobson L, Leudke C, Vogt SK, Schaefer ML, Dikkes P, Fukuda S, Sakai Y, Suda T \& Majzoub JA 2000

Corticotropin-releasing hormone links pituitary adrenocorticotropin gene expressiom and release during insufficiency. Journal of Clinical Investigation 105 1269-1277.

Nicholson WE \& Orth DN 1996 Preprothyrotropin-releasing hormone-(178-199) does not inhibit corticotropin release. Endocrinology 137 2171-2174.

Nillni EA, Aird F, Seidah NG, Todd RB \& Koenig JI 2001 Prepro TRH ${ }_{178-199}$ and two novel peptides $\left(\mathrm{pFQ}_{7}\right.$ and $\left.\mathrm{pSE}_{14}\right)$ derived from its processing, which are produced in the paraventricular nucleus of the rat hypothalamus. Endocrinology 142 896-906.

Redei E, Hilderbrand H \& Aird F 1995 Corticotropin release-inhibiting factor is preprothyrotropin-releasing hormone-(178-199). Endocrinology 136 3557-3563.

Redei E, Organ M \& Hart S 1999 Antidepressant-like properties of prepro-TRH 178-199: acute effects in the forced swim test. Neuroreport 10 3273-3276. 
Rittenhouse PA, Zorrilla EP, McGivern RF \& Redei E 1996 Restraint stress produces region specific changes in rat brain prepro-TRH 178-199, a proposed novel corticotropin release inhibiting factor. Society for Neuroscience Abstract 221341.

Roussel JP, Teresi S, Vaudry H \& Astier H 1994 A cryptic peptide of TRH prohormone inhibits TRH-induced GH release. Comptes Rendus de l'Academie des Sciences Serie III, Sciences de la Vie $\mathbf{3 1 7}$ 270-276.
Woods MD, Shipston MJ, Mullens EL \& Antoni FA 1992 Pituitary corticotroph tumor (AtT-20) cells as a model system for the study of early inhibition by glucocorticoids. Endocrinology 131 $2873-2880$.

Received 6 August 2001

Accepted 13 August 2001 